\title{
Article \\ Arities and Aritizabilities of First-Order Theories
}

\author{
Sergey V. Sudoplatov ${ }^{1 *(1)}$
}

1 Sobolev Institute of Mathematics, Academician Koptyug ave., 4, Novosibirsk, 630090, Russia; Novosibirsk State Technical University, K.Marx ave, 20, Novosibirsk, 630073, Russia; sudoplat@math.nsc.ru

* Correspondence: sudoplat@math.nsc.ru; Tel.: +7-383-329-7586

\begin{abstract}
We study and describe possibilities for arities of elementary theories and of their expansions. Links for arities with respect to Boolean algebras, to disjoint unions and to compositions of structures are shown. Arities and aritizabilities are semantically characterized. The dynamics for arities of theories is described.
\end{abstract}

Keywords: elementary theory; arity; expansion; aritizability

MSC: 03C07, 03C10, 03C68

\section{Introduction}

Arities of theories are important characteristics showing complexity measures of theories [1,2] and reducing all definable sets to definable ones generated by cylinders of special forms. It is closely linked with cylindric algebras reflecting semantically first-order calculi [3-7].

Special cases for arities of theories, especially binary, ternary and related ordered theories are studied in a series of papers including [8-14]. Structures and links with respect to binary formulas are investigated both in general case [15-18] and for a series of natural classes of theories [19-27].

In the present paper we adapt the general cylindric approach and describe semantically arities of theories, properties related to the $n$-arity and $n$-aritizability of theories and their dynamics.

The paper in organized as follows. In Section 1, we consider arities of formulae and arities of theories, describe possibilities of arities, describe arities for a series of natural theories, characterize the $\omega$-categoricity and the stability of $n$-ary theories. In Section 2, we introduce a series of notions for the aritizability of a theory, describe sufficient conditions and criteria for the aritizabilities in terms of Boolean algebras, disjoint unions and compositions. In Section 3, possibilities for aritizabilities are characterized semantically and the dynamics for arities of theories is described.

Throughout we consider complete first-order theories $T$.

\section{1. $n$-ary formulae and theories}

Definition [28]. A theory $T$ is said to be $\Delta$-based, where $\Delta$ is some set of formulae without parameters, if any formula of $T$ is equivalent in $T$ to a Boolean combination of formulae in $\Delta$.

For $\Delta$-based theories $T$, it is also said that $T$ has quantifier elimination or quantifier reduction up to $\Delta$.

Definition $[15,28]$. Let $\Delta$ be a set of formulae of a theory $T$, and $p(\bar{x})$ a type of $T$ lying in $S(T)$. The type $p(\bar{x})$ is said to be $\Delta$-based if $p(\bar{x})$ is isolated by a set of formulas $\varphi^{\delta} \in p$, where $\varphi \in \Delta, \delta \in\{0,1\}$.

The following lemma, being a corollary of Compactness Theorem, noticed in [28]. 
Lemma 1.1. A theory $T$ is $\Delta$-based if and only if, for any tuple $\bar{a}$ of any (some) weakly saturated model of $T$, the type $\operatorname{tp}(\bar{a})$ is $\Delta$-based.

Definition (cf. [1]). An elementary theory $T$ is called unary, or 1-ary, if any $T$ formula $\varphi(\bar{x})$ is $T$-equivalent to a Boolean combination of $T$-formulas, each of which is of one free variable, and of formulas of form $x \approx y$.

For a natural number $n \geq 1$, a formula $\varphi(\bar{x})$ of a theory $T$ is called $n$-ary, or an $n$-formula, if $\varphi(\bar{x})$ is $T$-equivalent to a Boolean combination of $T$-formulas, each of which is of $n$ free variables.

For a natural number $n \geq 2$, an elementary theory $T$ is called $n$-ary, or an $n$-theory, if any $T$-formula $\varphi(\bar{x})$ is $n$-ary.

A theory $T$ is called binary if $T$ is 2-ary, it is called ternary if $T$ is 3-ary, etc.

We will admit the case $n=0$ for $n$-formulae $\varphi(\bar{x})$. In such a case $\varphi(\bar{x})$ is just $T$-equivalent to a sentence $\forall \bar{x} \varphi(\bar{x})$.

If $T$ is a theory such that $T$ is $n$-ary and not $(n-1)$-ary then the value $n$ is called the arity of $T$ and it is denoted by $\operatorname{ar}(T)$. If $T$ does not have any arity we put $\operatorname{ar}(T)=\infty$.

Similarly, for a formula $\varphi$ of a theory $T$ we denote by $\operatorname{ar}_{T}(\varphi)$ the natural value $n$ if $\varphi$ is $n$-ary and not $(n-1)$-ary. If $\varphi$ does not any arity we $\operatorname{put}^{\operatorname{ar}_{T}}(\varphi)=\infty$. If a theory $T$ is fixed we write $\operatorname{ar}(\varphi)$ instead of $\operatorname{ar}_{T}(\varphi)$.

By the definition any $n$-theory is $\Delta_{n}$-based, where $\Delta_{n}$ consists of formulae with $n$ free variables and formulae of the form $x \approx y$. It implies that theories of $n$-element models $\mathcal{M}$ are $n$-ary and based by formulae describing these $n$-element structures and differences/coincidences of elements. formula:

Using Lemma 1.1 we obtain the following characterization for the $n$-arity of a

Proposition 1.2. A T-formula $\varphi(\bar{x})$ is not n-ary if and only if for any T-formulae $\psi_{i}\left(\bar{x}_{i}\right)$ with subtuples $\bar{x}_{i}$ of the tuple $\bar{x}$ having $l\left(\bar{x}_{i}\right)=n$ and $T \vdash \varphi(\bar{x}) \rightarrow \psi_{i}\left(\bar{x}_{i}\right)$, there exists a tuple $\bar{a} \in \mathcal{M} \models T$ such that $\mathcal{M} \models \psi_{i}\left(\bar{a}_{i}\right) \wedge \neg \varphi(\bar{a})$, where $\bar{a}_{i}$ is a subtuple of $\bar{a}$ consisting of substitutions of elements of $\bar{a}$ instead of correspondent elements of $\bar{x}_{i}$.

By the definition the notion of $n$-arity is local and reduced to finite sublanguages:

Proposition 1.3. A theory $T$ of a language $\Sigma$ is n-ary if and only if for any T-formula $\varphi(\bar{x})$ there is a finite sublanguage $\Sigma^{\prime} \subseteq \Sigma$ such that $T \vdash \varphi(\bar{x}) \leftrightarrow \psi(\bar{x})$, where $\psi(\bar{x})$ is a Boolean combination of $n$-formulae.

Proposition 1.4. If $\mathcal{M}$ is a n-element structure, for $n \in \omega$, then $\operatorname{ar}(\operatorname{Th}(\mathcal{M})) \leq n$.

Proof. Since $|M|=n$ each $\operatorname{Th}(\mathcal{M})$-formula $\varphi:=\varphi\left(x_{1}, \ldots, x_{m}\right)$ is $\operatorname{Th}(\mathcal{M})$-equivalent to a disjunction of substitutions of variables $x_{i_{1}}, \ldots, x_{i_{n}}$ instead of $x_{1}, \ldots, x_{m}$ into the formula $\varphi$, as required.

Remark 1.5. (cf. [4,7]) Since negations of formulas with $n$ free variables again have $n$ free variables, witnessing the $n$-arity of a formula it suffices to consider positive Boolean combinations of formulas with $n$ free variables, i.e., conjunctions and disjunctions of formulas with $n$ free variables.

Thus for the description of definable sets for models $\mathcal{M}$ of $n$-theories it suffices describe links between definable sets $A$ and $B$ for $n$-formulas $\varphi(\bar{x})$ and $\psi(\bar{y})$, respectively, and definable sets $C$ and $D$ for $\varphi(\bar{x}) \wedge \psi(\bar{y})$ and $\varphi(\bar{x}) \vee \psi(\bar{y})$, respectively.

If $\bar{x}=\bar{y}$ then $C=A \cap B$ and $D=A \cup B$, i.e., conjunctions and disjunctions work as set-theoretic intersections and unions.

If $\bar{x}$ and $\bar{y}$ are disjoint then $C=A \times B$ and $D=(A+B)_{\mathcal{M}} \rightleftharpoons\{\langle\bar{a}, \bar{b}\rangle \mid \bar{a} \in$ $A$ and $\bar{b} \in M$, or $\bar{a} \in M$ and $\bar{b} \in B$, i.e., $C$ is the Cartesian product of $A$ and $B$, and $D$ is the (generalized) Cartesian sum of $A$ and $B$ in the model $\mathcal{M}$.

If $\bar{x} \neq \bar{y}$, and $\bar{x}$ and $\bar{y}$ have common variables, then $C$ and $D$ are represented as a mixed product and a mixed sum, respectively, working partially as intersection and union, 
for common variables, and partially as Cartesian product and Cartesian sum, for disjoint variables.

If $\bar{x}$ and $\bar{y}$ consist of pairwise disjoint variables and $\bar{x} \subseteq \bar{y}$ and $\bar{x} \neq \bar{y}$ then for any formula $\varphi(\bar{x})$ the set of solution of the formula $\varphi(\bar{x}) \wedge(\bar{y} \approx \bar{y})$ in $\mathcal{M}$ is called a cylinder with respect to $M^{l(\bar{y})}$ and generated by the set of solutions $\varphi(\mathcal{M})$. In any case generating sets for cylinders coincide their projections, i.e., sets of solutions for formulas $\exists \bar{z} \varphi(\bar{x})$, where $\bar{z} \subset \bar{x}$.

Since $n$-formulae produce cylinders on Cartesian products of universes, definable sets of $n$-ary theories are composed by Boolean combinations of definable cylinders, i.e., of elements of cylindric algebras.

Definition (cf. [2]). For a natural number $n$, a theory $T$ is called $n$-transitive if each $n$-type $q\left(x_{1}, \ldots, x_{n}\right) \in S(T)$ is forced by its restriction to the empty language.

Proposition 1.6. If a theory $T$ is n-transitive and non- $(n+1)$-transitive then $T$ is not an n-theory.

Proof. Since $T$ is $n$-transitive, cylinders defined by $n$-formulae are reduced to the cylinders defined by the formulae for the empty language, i.e., they are defined by equalities and inequalities. As $T$ is not $(n+1)$-transitive then there is a $\varnothing$-definable set $X \subset M^{n+1}$ in a model $\mathcal{M} \models T$ which in not reduced to the cylinders defined by the formulae for the empty language. It means that a formula $\varphi\left(x_{1}, \ldots, x_{n+1}\right)$ defining $X$ is not $T$-equivalent to $n$-formulae. Thus $T$ is not an $n$-theory, as required.

Clearly, generic constructions [15,29] allow to produce, for each $n \geq 1, n$-transitive and non- $(n+1)$-transitive theories with unique $(n+1)$-ary predicates and having quantifier elimination.

For instance, the theory $T$ of structure $\mathcal{M}=\left\langle\{a, b, c, d\} ; R^{(3)}\right\rangle$ with the ternary relation $R=\{(a, b, c),(b, a, d),(b, c, d),(c, b, a),(a, c, d),(c, a, b),(c, d, a),(d, c, b),(d, a, b),(a, d, c)$, $(b, d, a),(d, b, c)\}$ has quantifier elimination, is 2-transitive, not 3-transitive, and thus $\operatorname{ar}(T)=3$.

This example can be naturally spread for $n$-ary relations. In view of Proposition 1.6 it implies the following:

Corollary 1.7. For any natural $n \geq 1$ there is a theory $T_{n}$ with $\operatorname{ar}\left(T_{n}\right)=n$.

The following examples illustrate values $\operatorname{ar}(T)=n$.

Example 1.8. [30] For any theory $T_{f}$ of an unar, i.e., of one unary operation $f$, $\operatorname{ar}(T) \leq 2$. There are both theories $T_{f_{1}}$ with $\operatorname{ar}\left(T_{f_{1}}\right)=1$ and theories $T_{f_{2}}$ with $\operatorname{ar}\left(T_{f_{2}}\right)=2$. For instance, $f_{1}$ can be taken identical, and $f_{2}$ - a successor function on at least 3 -element set.

Example 1.9. [30] For any theory $T_{\Gamma}$ of an acyclic graph $\Gamma$ with unary predicates, $\operatorname{ar}\left(T_{\Gamma}\right) \leq 2$.

Example 1.10. Let $E$ be the following equivalence relation on the set $\mathbf{R}^{n}$ :

$$
\left\{(M, N) \mid M\left(x_{1}, \ldots, x_{n}\right), N\left(y_{1}, \ldots, y_{n}\right) \in \mathbf{R}^{n}, x_{1}^{2}+\ldots+x_{n}^{2}=y_{1}^{2}+\ldots+y_{n}^{2}\right\} .
$$

Equivalence classes for the concentric spheres in $\mathbf{R}^{n}$ can not be reconstructed via cylinders defined by projections which form concentric balls and circles. The homogeneity of equivalence classes implies that each formula in the language $\langle E\rangle$ is reduced to a Boolean combination of $2 n$-formulas. Thus $\operatorname{Th}(\langle\mathbf{R}, E\rangle)$ is a $2 n$-theory which is not an $(2 n-1)$-theory.

Adding a disjoint unary predicate $P$ and a bijection $f$ between the set of spheres and $P$ we obtain names for spheres and an additional coordinate for generating formulas for a basedness. Thus we form a $(2 n+1)$-theory which is not an $2 n$-theory.

Hence all possibilities for $\operatorname{ar}(T)=n$ are realized. 
Example 1.11. Taking a non-degenerated algebraic surface at $\mathbf{R}^{n}$ which is not reduced to cylinders we obtain a defining formula $\varphi(\bar{x}), l(\bar{x})=n+1$, which is $(n+1)$ formula and not an $n$-formula. In particular, non-degenerated non-cylindrical surfaces of the second order in $\mathbf{R}^{3}$ are defined by formulas $\varphi$ with $\operatorname{ar}(\varphi)=3$. For instance, taking the formula $x^{2}+y^{2}+z^{2}=1$ for the sphere $S$ we obtain projections $x^{2}+y^{2} \leq 1, x^{2}+z^{2} \leq 1$, $y^{2}+z^{2} \leq 1$ which can not allow to reconstruct $S$ by their Boolean combinations.

Example 1.12. Recall $[8,11,12]$ that a circular, or cyclic order relation is described by a ternary relation $K_{3}$ satisfying the following conditions:

(co1) $\forall x \forall y \forall z\left(K_{3}(x, y, z) \rightarrow K_{3}(y, z, x)\right)$;

(co2) $\forall x \forall y \forall z\left(K_{3}(x, y, z) \wedge K_{3}(y, x, z) \leftrightarrow x=y \vee y=z \vee z=x\right)$;

(co3) $\forall x \forall y \forall z\left(K_{3}(x, y, z) \rightarrow \forall t\left[K_{3}(x, y, t) \vee K_{3}(t, y, z)\right]\right)$;

(co4) $\forall x \forall y \forall z\left(K_{3}(x, y, z) \vee K_{3}(y, x, z)\right)$.

Clearly, $\operatorname{ar}\left(K_{3}(x, y, z)\right)=3$ if the relation has at least three element domain. Hence, theories with infinite circular order relations are at least 3-ary.

The following generalization of circular order produces a $n$-ball, or $n$-spherical, or $n$-circular order relation, for $n \geq 4$, which is described by a $n$-ary relation $K_{n}$ satisfying the following conditions:

(nbo1) $\forall x_{1}, \ldots, x_{n}\left(K_{n}\left(x_{1}, x_{2}, \ldots, x_{n}\right) \rightarrow K_{n}\left(x_{2}, \ldots, x_{n}, x_{1}\right)\right)$;

(nbo2) $\forall x_{1}, \ldots, x_{n}\left(K_{n}\left(x_{1}, \ldots, x_{i}, x_{i+1}, \ldots, x_{n}\right) \wedge\right.$

$$
\left.\wedge K_{n}\left(x_{1}, \ldots, x_{i+1}, x_{i}, \ldots, x_{n}\right) \leftrightarrow \bigvee_{i=1}^{n-1} x_{i}=x_{i+1}\right)
$$

(nbo3) $\forall x_{1}, \ldots, x_{n}\left(K_{n}\left(x_{1}, \ldots, x_{n}\right) \rightarrow \forall t\left[K_{n}\left(x_{1}, \ldots, x_{n-1}, t\right) \vee K_{n}\left(t, x_{2}, \ldots, x_{n}\right)\right]\right)$; (nbo4) $\forall x_{1}, \ldots, x_{n}\left(K_{n}\left(x_{1}, \ldots, x_{i}, x_{i+1}, \ldots, x_{n}\right) \vee K_{n}\left(x_{1}, \ldots, x_{i+1}, x_{i}, \ldots, x_{n}\right)\right), i<n$.

Clearly, $\operatorname{ar}\left(K_{n}\left(x_{1}, \ldots, x_{n}\right)\right)=n$ if the relation has at least $n$-element domain. Thus, theories with infinite $n$-ball order relations are at least $n$-ary.

Theorem 1.13. An n-ary theory $T$ is $\omega$-categorical if and only if there are finitely many $T$-non-equivalent formulas with $n$ free variables.

Proof. If $T$ is $\omega$-categorical then by Ryll-Nardzewski Theorem there are finitely many $T$-non-equivalent formulas with $m$ free variables for every $m$, in particular, for $m=n$. Conversely, we again apply Ryll-Nardzewski Theorem showing that are finitely many $T$-non-equivalent formulas with $m$ free variables for every $m$. If $m \leq n$ then there are finitely many $T$-non-equivalent formulas with $m$ free variables by the monotony of this property with respect to the number of free variables. If $m>n$ then by the $n$-arity of $T$ each $T$-formula $\varphi\left(x_{1}, \ldots, x_{m}\right)$ is $T$-equivalent to a Boolean combination of formulas with $n$ free variables. Since there are finitely many $T$-non-equivalent possibilities for these formulas, Boolean combinations produce finitely many possibilities, too. As there are finitely many $T$-non-equivalent formulas with $m$ free variables for every $m$ then $T$ is $\omega$-categorical by Ryll-Nardzewski Theorem, as required.

Recall [31] that a formula $\varphi(\bar{x}, \bar{y})$ of a theory $T$ is stable if there are no tuples $\bar{a}_{n}, \bar{b}_{n}$, $n \in \omega$, such that $=\varphi\left(\bar{a}_{i}, \bar{b}_{j}\right) \Leftrightarrow i \leq j$. The theory $T$ is called stable if every $T$-formula is stable.

In [32], it was shown that any Boolean combination of stable formulas is again a stable formula. Thus, using the definition of $n$-ary theory we obtain the following:

Theorem 1.14. An n-ary theory $T$ is stable if and only if each $T$-formula with $n$ free variables is stable. 


\section{Aritizable formulae and theories}

Definition. A $T$-formula $\varphi(\bar{x})$ is called n-expansible, or n-arizable, or $n$-aritizable, if $T$ has an expansion $T^{\prime}$ such that $\varphi(\bar{x})$ is $T^{\prime}$-equivalent to a Boolean combination of $T^{\prime}$-formulas with $n$ free variables.

A theory $T$ is called $n$-expansible, or $n$-arizable, or $n$-aritizable, if there is an $n$-ary expansion $T^{\prime}$ of $T$.

A theory $T$ is called arizable or aritizable, if $T$ is $n$-aritizable for some $n$.

A 1-aritizable theory is called unary-able, or unary-tizable. A 2-aritizable theory is called binary-tizable or binarizable, a 3-aritizable theory is called ternary-tizable or ternarizable, etc.

By the definition any $n$-theory is $n$-expansible, by itself, and if $T$ is $n$-expansible then $T$ is $m$-expansible for each $m>n$.

Besides each formula of an $n$-expansible theory is $n$-expansible, too, but not vice versa in the following sense: if each formula of a theory $T$ is $n$-expansible, it can not guarantee that a resulting expansion $T^{\prime}$, witnessing that $n$-expansibility, is coordinated enough such that it is $n$-ary or at least $n$-expansible.

Proposition 2.1. Any theory of a finite structure $\mathcal{M}$ is binarizable.

Proof. Let $M=\left\{a_{1}, \ldots, a_{m}\right\}$. For any pair $\left\langle a_{i}, a_{j}\right\rangle, i, j \leq m$, we introduce new binary singleton predicate $B_{i, j}=\left\{\left\langle a_{i}, a_{j}\right\rangle\right\}$. We denote the resulted expansion of $\mathcal{M}$ by $\mathcal{M}^{\prime}$, and the theory $\operatorname{Th}\left(\mathcal{M}^{\prime}\right)$ expanding given theory $T=\operatorname{Th}(\mathcal{M})$ by $T^{\prime}$. Now an arbitrary $T^{\prime}$-formula $\varphi(\bar{x})$, with $\bar{x}=\left\langle x_{1}, \ldots, x_{n}\right\rangle$, has finitely many solutions $\bar{b}=\left\langle a_{k_{1}}, \ldots, a_{k_{n}}\right\rangle$ in $\mathcal{M}^{\prime}$. We collect these solutions into a set $Z$. Without loss of generality $Z \neq \varnothing$ since for $Z=\varnothing, T \vdash \varphi(\bar{x}) \leftrightarrow \neg x_{i} \approx x_{i}$ for any $x_{i}$. Now the formula $\varphi(\bar{x})$ is $T^{\prime}$-equivalent to the following Boolean combination of binary formulae:

$$
\bigvee_{\left\langle a_{k_{1}}, \ldots, a_{k_{n}}\right\rangle \in Z} \bigwedge_{i, j \leq n} B_{k_{i}, k_{j}}\left(x_{i}, x_{j}\right),
$$

as required.

Remark 2.2. If $m, n \in \omega \backslash\{0\}$ and $M$ is an $m$-element set, then $M^{n}$ has $2^{\left(m^{n}\right)}$ subsets producing distinct $n$-ary predicates $Q_{i}, i<2^{\left(m^{n}\right)}$. Since by Stone Theorem any finite Boolean has $2^{l}$ elements with $l$ generators, there are $m^{n}$ independent predicates $Q_{j}^{\prime}$ whose Boolean combinations produce all these predicates. Taking a quantifier free formula $\varphi\left(x_{1}, \ldots, x_{k}\right)$, for $k \geq n$, composed by these independent predicates $Q_{j}^{\prime}$ and having a perfect disjunctive normal form we obtain $A_{k}^{n} \cdot 2^{m^{n}}$ possibilities for disjunctive members, where $A_{k}^{n}$ is used to calculate the number of choice of $n$ variables among $x_{1}, \ldots, x_{k}$ and there are $2^{m^{n}}$ possibilities for positive and negative entries of $Q_{j}^{\prime}$. Now there are $2^{A_{k}^{n} \cdot 2^{m^{n}}}$ possibilities for $\varphi\left(x_{1}, \ldots, x_{k}\right)$, big enough. At the same time, using the arguments for Proposition 2.1 we can obtain all definable subsets of $M^{n}$ just using $m^{2}$ singleton binary relations.

Applying arguments for Proposition 2.1 we immediately obtain the following:

Proposition 2.3. Any formula of a theory having finitely many solutions is binarizable.

Proposition 2.1 can be strengthened as follows:

Proposition 2.4. Any theory of a finite structure $\mathcal{M}$ is unary-tizable.

Proof. Let $M=\left\{a_{1}, \ldots, a_{m}\right\}$. For element $a_{i}, i \leq m$, we introduce new unary singleton predicate $U_{i}=\left\{a_{i}\right\}$. We denote the resulted expansion of $\mathcal{M}$ by $\mathcal{M}^{\prime}$, and the theory $\operatorname{Th}\left(\mathcal{M}^{\prime}\right)$ expanding given theory $T=\operatorname{Th}(\mathcal{M})$ by $T^{\prime}$. Now an arbitrary $T^{\prime}$-formula $\varphi(\bar{x})$, with $\bar{x}=\left\langle x_{1}, \ldots, x_{n}\right\rangle$, has finitely many solutions $\bar{b}=\left\langle a_{k_{1}}, \ldots, a_{k_{n}}\right\rangle$ in $\mathcal{M}^{\prime}$. We collect these solutions into a set $Z$. Without loss of generality $Z \neq \varnothing$. Now the formula 
$\varphi(\bar{x})$ is $T^{\prime}$-equivalent to the following Boolean combination of formulae each of which with one free variable:

$$
\bigvee_{\left\langle a_{k_{1}}, \ldots, a_{k_{n}}\right\rangle \in Z} \bigwedge_{i, j \leq n} U_{k_{i}}\left(x_{i}\right),
$$

as required.

Thus $|\mathcal{M}|$-many unary predicates produce a unary expansion of the theory $\operatorname{Th}(\mathcal{M})$ with finite $\mathcal{M}$. Besides using the proof of Proposition 2.4 we have:

Proposition 2.5. Any formula of a theory having finitely many solutions is unary-tizable.

Remark 2.6. By the definition for any natural $n$ both $n$-ary formulae and $n$-aritizable formulae of a fixed theory $T$ are closed under Boolean combinations. Therefore taking a model $\mathcal{M}=T$ and collecting in sets $\operatorname{BA}_{k n}(\mathcal{M})$ and $\operatorname{BA}_{k n}^{\prime}(\mathcal{M})$ definable sets which are defined by $n$-ary, respectively, $n$-aritizable formulae with $k$ free variables we obtain Boolean algebras $\mathcal{B} \mathcal{A}_{k n}(\mathcal{M})$ and $\mathcal{B} \mathcal{A}_{k n}^{\prime}(\mathcal{M})$ of these definable sets.

Clearly, $\mathcal{B} \mathcal{A}_{k n}(\mathcal{M}) \subseteq \mathcal{B} \mathcal{A}_{k n}^{\prime}(\mathcal{M})$, and the equality $\mathcal{B} \mathcal{A}_{k n}(\mathcal{M})=\mathcal{B} \mathcal{A}_{k n}^{\prime}(\mathcal{M})$ means that any $n$-aritizable formula $\varphi\left(x_{1}, \ldots, x_{n}\right)$ of $T$ is already $n$-ary.

In view of Proposition 2.5 the algebra $\mathrm{BA}_{k n}^{\prime}(\mathcal{M})$ satisfies the following condition: if $X$ and $Y$ are $\varnothing$-definable subsets of $M^{k}$ with finite symmetric difference $X \div Y$ then $X \in \mathrm{BA}_{k n}^{\prime}(\mathcal{M})$ iff $Y \in \mathrm{BA}_{k n}^{\prime}(\mathcal{M})$. At the same time $\mathrm{BA}_{k n}(\mathcal{M})$ can be not closed under finite symmetric difference since, for instance, there are theories of finite structures which are not $n$-ary but by Proposition 2.4 all theories of finite structures are unary-tizable.

The Boolean algebras $\mathcal{B} \mathcal{A}_{k n}(\mathcal{M})$ and $\mathcal{B} \mathcal{A}_{k n}^{\prime}(\mathcal{M})$ have extensions $\mathcal{B} \mathcal{A}_{k}(\mathcal{M})$ and $\mathcal{B} \mathcal{A}_{k}^{\prime}(\mathcal{M})$, respectively, consisting of definable sets for $n$-ary $/ n$-aritizable formulae with $m$ free variables, for some $n$. Clearly, both $\mathcal{B} \mathcal{A}_{k}(\mathcal{M})$ and $\mathcal{B} \mathcal{A}_{k}^{\prime}(\mathcal{M})$ equal the Boolean algebra $\mathcal{B}_{k}(\mathcal{M})$ of all $\varnothing$-definable subsets of $M^{k}$. Both these inclusions $\mathcal{B A}_{k n}(\mathcal{M}) \subseteq$ $\mathcal{B}_{k}(\mathcal{M})$ and $\mathcal{B} \mathcal{A}_{k n}^{\prime}(\mathcal{M}) \subseteq \mathcal{B}_{k}(\mathcal{M})$ can be proper for $k>n$.

It was noticed above that aritizabilities of separated formulae of a theory can not guarantee that witnesses of these aritizabilities produce a $n$-ary theory, the possibility of coordinated expansion of aritizable formulae is necessary. We denote by $\mathcal{B} \mathcal{A}_{k n}^{\prime \prime}(\mathcal{M})$ the Boolean algebra $\mathcal{B} \mathcal{A}_{k n}^{\prime}(\mathcal{M})$ with a coordinated $n$-ary expansion for all $n$-aritizable formulae with $k$ free variables.

Using Remark 2.6 we have the following characterizations of $n$-arity and of $n$ aritizability of a theory $T$ in terms of Boolean algebras of a model for $T$. hold:

Proposition 2.7. For any theory $T$, its model $\mathcal{M}$, and $n \in \omega$ the following conditions

(1) $T$ is $n$-ary iff $\mathcal{B A}_{k n}(\mathcal{M})=\mathcal{B}_{k}(\mathcal{M})$ for each $k>n$;

(1) $T$ is n-aritizable iff $\mathcal{B} \mathcal{A}_{k n}^{\prime \prime}(\mathcal{M})=\mathcal{B}_{k}(\mathcal{M})$ for each $k>n$.

Definition. [33] The disjoint union $\bigsqcup_{n \in \omega} \mathcal{M}_{n}$ of pairwise disjoint structures $\mathcal{M}_{n}$ for pairwise disjoint predicate languages $\Sigma_{n}, n \in \omega$, is the structure of language $\bigcup_{n \in \omega} \Sigma_{n} \cup$ $\left\{P_{n}^{(1)} \mid n \in \omega\right\}$ with the universe $\bigsqcup_{n \in \omega} M_{n}, P_{n}=M_{n}$, and interpretations of predicate symbols in $\Sigma_{n}$ coinciding with their interpretations in $\mathcal{M}_{n}, n \in \omega$. The disjoint union of theories $T_{n}$ for pairwise disjoint languages $\Sigma_{n}$ accordingly, $n \in \omega$, is the theory

$$
\bigsqcup_{n \in \omega} T_{n} \rightleftharpoons \operatorname{Th}\left(\bigsqcup_{n \in \omega} \mathcal{M}_{n}\right),
$$

where $\mathcal{M}_{n} \models T_{n}, n \in \omega$. Taking empty sets instead of some structures $\mathcal{M}_{k}$ we obtain disjoint unions of finitely many structures and theories. In particular, we have the disjoint unions $\mathcal{M}_{0} \sqcup \ldots \sqcup \mathcal{M}_{n}$ and their theories $T_{0} \sqcup \ldots \sqcup T_{n}$.

Clearly, disjoint unions of theories does not depend on choice of correspondent disjoint unions of their models. Besides, disjoint unions $\bigsqcup_{n \in \omega} T_{n}$ are based by the unions 
of the basing sets $\Delta_{n}$ for $T_{n}$ and by the formulae of the form $P_{n}(x)$. Thus we have the following:

Theorem 2.8. 1. For any theories $T_{m}, m \in \omega$, and their disjoint union $\bigsqcup_{m \in \omega} T_{m}$, all $T_{m}$ are n-theories iff $\bigsqcup_{m \in \omega} T_{m}$ is an n-theory, moreover, $\operatorname{ar}\left(\bigsqcup_{m \in \omega} T_{m}\right)=\max \left\{\operatorname{ar}\left(T_{m}\right) \mid m \in \omega\right\}$.

2. For any theories $T_{m}, m \in \omega$, and their disjoint union $\bigsqcup_{m \in \omega} T_{m}$, all $T_{m}$ are n-aritizable iff $\bigsqcup_{m \in \omega} T_{m}$ is n-aritizable.

Definition [27]. Let $\mathcal{M}$ and $\mathcal{N}$ be structures of relational languages $\Sigma_{\mathcal{M}}$ and $\Sigma_{\mathcal{N}}$ respectively. We define the composition $\mathcal{M}[\mathcal{N}]$ of $\mathcal{M}$ and $\mathcal{N}$ satisfying the following conditions:

1) $\Sigma_{\mathcal{M}[\mathcal{N}]}=\Sigma_{\mathcal{M}} \cup \Sigma_{\mathcal{N}}$ tively;

2) $M[N]=M \times N$, where $M[N], M, N$ are universes of $\mathcal{M}[\mathcal{N}], \mathcal{M}$, and $\mathcal{N}$ respec-

3) if $R \in \Sigma_{\mathcal{M}} \backslash \Sigma_{\mathcal{N}}, \mu(R)=n$, then $\left(\left(a_{1}, b_{1}\right), \ldots,\left(a_{n}, b_{n}\right)\right) \in R_{\mathcal{M}[\mathcal{N}]}$ if and only if $\left(a_{1}, \ldots, a_{n}\right) \in R_{\mathcal{M}}$;

4) if $R \in \Sigma_{\mathcal{N}} \backslash \Sigma_{\mathcal{M}}, \mu(R)=n$, then $\left(\left(a_{1}, b_{1}\right), \ldots,\left(a_{n}, b_{n}\right)\right) \in R_{\mathcal{M}[\mathcal{N}]}$ if and only if $a_{1}=\ldots=a_{n}$ and $\left(b_{1}, \ldots, b_{n}\right) \in R_{\mathcal{N}}$;

5) if $R \in \Sigma_{\mathcal{M}} \cap \Sigma_{\mathcal{N}}, \mu(R)=n$, then $\left(\left(a_{1}, b_{1}\right), \ldots,\left(a_{n}, b_{n}\right)\right) \in R_{\mathcal{M}[\mathcal{N}]}$ if and only if $\left(a_{1}, \ldots, a_{n}\right) \in R_{\mathcal{M}}$, or $a_{1}=\ldots=a_{n}$ and $\left(b_{1}, \ldots, b_{n}\right) \in R_{\mathcal{N}}$.

The composition $\mathcal{M}[\mathcal{N}]$ is called e-definable, or equ-definable, if $\mathcal{M}[\mathcal{N}]$ has an $\varnothing$ definable equivalence relation $E$ whose $E$-classes are universes of the copies of $\mathcal{N}$ forming $\mathcal{M}[\mathcal{N}]$. If the equivalence relation $E$ is fixed, the $e$-definable composition is called $E$ definable.

Using a nice basedness of E-definable compositions $T_{1}\left[T_{2}\right]$ (see [27]) till the formulas of form $E(x, y)$ and generating formulas for $T_{1}$ and $T_{2}$ we have the following:

Theorem 2.9. 1. For any theories $T_{1}$ and $T_{2}$ and their E-definable composition $T_{1}\left[T_{2}\right]$, $T_{1}$ and $T_{2}$ are $n$-theories, for $n \geq 2$, iff $T_{1}\left[T_{2}\right]$ is an $n$-theory, moreover, $\operatorname{ar}\left(T_{1}\left[T_{2}\right]\right)=$ $\max \left\{\operatorname{ar}\left(T_{1}\right), \operatorname{ar}\left(T_{2}\right)\right\}$, if models of $T_{1}$ and of $T_{2}$ have at least two elements, and $\operatorname{ar}\left(T_{1}\left[T_{2}\right]\right)=$ $\max \left\{\operatorname{ar}\left(T_{1}\right), \operatorname{ar}\left(T_{2}\right), 2\right\}$, if a model of $T_{1}$ or $T_{2}$ is a singleton.

2. For any theories $T_{1}$ and $T_{2}$ and their E-definable composition $T_{1}\left[T_{2}\right], T_{1}$ and $T_{2}$ are n-aritizable iff $T_{1}\left[T_{2}\right]$ is n-aritizable.

Applying Proposition 2.4 and Theorem 2.9 we immediately obtain:

Corollary 2.10. If each of theories $T_{1}$ and $T_{2}$ is a theory of a finite structure, or of an infinite structure and n-arizable, then their E-definable composition $T_{1}\left[T_{2}\right]$ is n-aritizable.

\section{Unary-tizable, binarizable and aritizable theories, their definable sets and dynamics}

Let $T$ be a theory with a unary expansion $T^{\prime}$. Since unary formulas $\varphi(x)$ and $\psi(y)$ have either equal or disjoint free variables we do not have essential mixed sums and mixed products forming definable sets for a model $\mathcal{M}$ of $T^{\prime}$, i.e., all definable sets are formed using unions, intersections, Cartesian sums and Cartesian products of definable subsets of $M$, without parameters.

Conversely, having a system of definable sets formed by unions, intersections, Cartesian sums and Cartesian products of subsets of $M$, we can introduce names for these subsets and generate, using this introduced language, all given definable sets.

Thus we obtain the following characterization for the unary-tizability of a theory in terms of definable sets:

Theorem 3.1. A theory $T$ is unary-tizable if and only if for any (some) model $\mathcal{M}$ of $T$ any definable set is formed by unions, intersections, Cartesian sums and Cartesian products of subsets of $M$. 
Similarly, all definable sets of binarizable theories are generated by unions, intersections, Cartesian sums and Cartesian products of subsets of $M^{2}$, extended by mixed sums and mixed products of these subsets and their combinations:

Theorem 3.2. A theory $T$ is binarizable if and only if for any (some) model $\mathcal{M}$ of $T$ any $\varnothing$-definable set is formed by unions, intersections, Cartesian sums, Cartesian products, mixed sums and mixed products of subsets of $M^{2}$.

By Theorem 3.2 definable sets of binarizable theories are generated by combinations of 3-dimensional cylinders with two-dimensional generators.

Theorems 3.1 and 3.2 admit the following natural generalizations based on $(n+1)$ dimensional cylinders with $n$-dimensional generators.

Theorem 3.3. A theory $T$ is $n$-aritizable, for $n \geq 1$, if and only if for any (some) model $\mathcal{M}$ of $T$ any $\varnothing$-definable set is formed by unions, intersections, Cartesian sums, Cartesian products, mixed sums and mixed products of subsets of $M^{n}$.

Theorem 3.4. A theory $T$ is aritizable if and only if for any (some) model $\mathcal{M}$ of $T$ any $\varnothing$-definable set is formed by unions, intersections, Cartesian sums, Cartesian products, mixed sums and mixed products of subsets of $M^{n}$, for some $n$.

Remark 3.5. Using Theorem 3.3 one can form a definable subset $X \subset M^{k}$ of an infinite model $\mathcal{M}$ of a $n$-theory, for $k>n$, such that $X$ has an infinite complement and each projection of $X$ equals $M^{m}$ for some $m<n$. It implies that Boolean combinations of these projections can not reconstruct $X$. Thus the structure $\langle M, X\rangle$ has a $n$-expansible $k$-theory.

Definition. A $T$-formula $\varphi(\bar{x})$ is called constantizable if $T$ has an expansion $T^{\prime}$ such that $\varphi(\bar{x})$ is $T^{\prime}$-equivalent to a Boolean combination of formulae of forms $x \approx y$ and $x \approx c$ with variables $x, y$ and constants $c$, i.e., $T$ has an expansion $T^{\prime \prime}$ such that $\varphi(\bar{x})$ is $T^{\prime}$-equivalent to a Boolean combination of formulae of forms $x \approx y$ and of formulae of unary singleton predicates whose solutions consist of constants.

A theory $T$ is called constantizable if any $T$-formula $\varphi(\bar{x})$ is constantizable.

By the definition any constantizable theory is unary-tizable, but not vice versa, as the following assertions show.

Proposition 3.6. A formula $\varphi(\bar{x})$ of a theory $T$ is constantizable iff $\varphi(\bar{x})$ is T-equivalent to a Boolean combination of formulae of form $x \approx y$ and formulae with finitely many solutions.

Proof. Let $\varphi(\bar{x})$ be a constantizable formula. Since $\varphi(\bar{x})$ is unary-tizable we can divide $\varphi(\bar{x})$ in some expansion of $T$ onto cases with distinct/equal values for free variables. Thus without loss of generality $\varphi(\bar{x})$ is a Boolean combination of formulae $\left(x_{i} \approx x_{j}\right)^{\delta}$ and $\psi\left(x_{i}\right)$, for $x_{i}, x_{j} \in \bar{x}, i \neq j, \delta \in\{0,1\}$, written in a disjunctive normal form. Since $\varphi(\bar{x})$ is constantizable, its definable set $A$ in a model of $T$ is represented by a Boolean combination of cylinders for $x \approx y$ and $x \approx c$. Thus, $\varphi(\bar{x})$ is $T$-equivalent to a Boolean combination of formulae of form $x \approx y$ and formulae with finitely many solutions.

Now let $\varphi(\bar{x})$ is T-equivalent to a Boolean combination of formulae of form $x \approx y$ and formulae $\psi(\bar{x})$ with finitely many solutions. We may assume that $\varphi(\bar{x})$ consistent and represented as a disjunctive normal form. We collect in a set $Z$ all finite sets of solutions for formulae $\psi(\bar{x})$. Now for the finite set $\cup Z$ we apply the construction for Proposition 2.4 reducing the set $Z^{\prime}$ of all coordinates for tuples in $\cup Z$ to unary singleton predicates and so to the formulae $x \approx c$, for $c \in Z^{\prime}$. It implies that a Boolean combination of these formulae and formulae of the form $x \approx y$ is equivalent to $\varphi(\bar{x})$, i.e., $\varphi(\bar{x})$ is constantizable, as required.

Proposition 3.6 immediately implies:

Corollary 3.7. A theory $T$ is constantizable iff each $T$-formula is T-equivalent to a Boolean combination of formulae of form $x \approx y$ and formulae with finitely many solutions. 
Definition [34]. A theory $T$ is called strongly minimal if for any formula $\varphi(x, \bar{a})$ of language obtained by adding parameters of $\bar{a}$ (in some model $\mathcal{M} \models T$ ) to the language of $T$, either $\varphi(x, \bar{a})$, or $\neg \varphi(x, \bar{a})$ has finitely many solutions.

Using Corollary 3.7 we obtain:

Corollary 3.8. Any constantizable theory is strongly minimal.

Now we consider some dynamics of arities of theories under expansions. Since the property of non- $n$-arizability forbids $n$-ary expansions it suffices to study possibilities for expansions of $n$-aritizable theories.

Proposition 3.9. A theory $T$ has a non-aritizable expansion iff $T$ has an infinite model.

Proof. If $T$ has an infinite model there are expansions $T^{\prime}$ of $T$ collecting, for instance, examples 1.10, 1.11, 1.12 forbidding $n$-arity for each $n$. Thus $T^{\prime}$ is not aritizable.

Conversely, if $T$ has an finite model then each expansion $T^{\prime}$ of $T$ has a finite model producing aritizability of $T^{\prime}$ by Proposition 2.4 , as required.

Using examples above we observe that for each natural $n \geq 1$ there are theories $T_{n}$ with $\operatorname{ar}\left(T_{n}\right)=n$ and finite models. Thus there are theories $T_{k n}$ with $\operatorname{ar}\left(T_{k n}\right)=k$ and $\operatorname{ar}\left(T_{k n}^{\prime}\right)=n$ for some expansions $T_{k n}^{\prime}$ of $T_{k n}$.

Besides, for each natural $n \geq 1$ there are:

1) theories $T_{n, \infty}$ with $\operatorname{ar}\left(T_{n, \infty}\right)=n$ and $\operatorname{ar}\left(T_{n, \infty}^{\prime}\right)=\infty$ for some expansions $T_{n, \infty}^{\prime}$ of $T_{n, \infty}$ : it suffices to expand a $n$-ary theory with infinite models by new predicates forbidding the $k$-aritizability for each $k>n$;

2) theories $T_{\infty, n}$ with $\operatorname{ar}\left(T_{\infty, n}\right)=\infty$ and $\operatorname{ar}\left(T_{\infty, n}^{\prime}\right)=n$ for some expansions $T_{\infty, n}^{\prime}$ of $T_{\infty, n}$ : it suffices to expand an $n$-aritizable theory which is not $m$-ary for any $m$ till a $n$-theory.

Thus the arities can be freely increased and decreased and we obtain the following:

Theorem 3.10. For any $\mu, v \in(\omega \backslash\{0\}) \cup\{\infty\}$ there is a theory $T_{\mu, v}$ and its expansion $T_{\mu, v}^{\prime}$ such that $\operatorname{ar}\left(T_{\mu, v}\right)=\mu$ and $\operatorname{ar}\left(T_{\mu, v}^{\prime}\right)=v$.

\section{Conclusion}

We considered possibilities for arities of theories and their dynamics, reductions of formulas to ones of special forms as well as definable sets connected with these reductions. It can be used both for databases, simplifying them to ones with bounded dimensions, for geometric objects represented as finite combinations of cylinders, and for cryptographic constructions representing complicated configurations by simpler ones. It would be interesting to describe values of arities and aritizabilities for natural classes of theories.

\section{Patents}

Funding: The work of the author was carried out in the framework of the State Contract of the Sobolev Institute of Mathematics, Project No. FWNF-2022-0012 (Section 1), and of Russian Scientific Foundation, Project No. 22-21-00044 (Sections 2 and 3).

Conflicts of Interest: The author declares no conflict of interest.

\section{References}

1. Sudoplatov, S.V. On a certain complexity estimate in graph theory. Siberian Mathematical. Journal 1996, 37:3, 614-671.

2. Sudoplatov, S.V. Transitive arrangements of algebraic systems. Siberian Mathematical. Journal 1999, 40:6, $1142-1145$.

3. Mal'tsev A.I. Algebraic systems; Nauka: Moscow, 1970.

4. Henkin, L., Monk, J.D., Tarski, A. Cylindric Algebras. Part 1; : Amsterdam, London, 1971.

5. Henkin, L., Monk, J.D., Tarski, A. Cylindric Set Algebras. Lecture Notes in Mathematics. Vol. 883; Springer-Verlag: Berlin, Heidelberg, New York, 1981.

6. Henkin, L., Monk, J.D., Tarski, A. Cylindric Algebras. Part 2. Studies in Logic and Foundations of Mathematics. Vol. 115; : Amsterdam, London, Oxford, 1985. 
7. Imieliński, T., Lipski, Jr.W. The relational model of data and cylindric algebras. Journal of Computer and System Sciences 1984, 28:1, 80-102.

8. Kulpeshov, B.Sh., Macpherson, H.D. Minimality conditions on circularly ordered structures. Mathematical Logic Quarterly 2005, 51:4, 377-399.

9. Baizhanov, B.S., Kulpeshov, B.Sh. On behaviour of 2-formulas in weakly o-minimal theories. Mathematical Logic in Asia, Proceedings of the 9th Asian Logic Conference. Eds.: S. Goncharov, R. Downey, H. Ono; World Scientific: Singapore, 2006, 31-40.

10. Kulpeshov, B.Sh. Criterion for binarity of $\aleph_{0}$-categorical weakly o-minimal theories. Annals of Pure and Applied Logic 2007, 45 , 354-367.

11. Altaeva, A.B., Kulpeshov, B.Sh. On almost binary weakly circularly minimal structures. Bulletin of Karaganda University, Mathematics 2015, 78:2, 74-82.

12. Kulpeshov, B.Sh. On almost binarity in weakly circularly minimal structures. Eurasian Mathematical Journal 2016, 7:2, 38-49.

13. Altaeva, A.B., Kulpeshov, B.Sh. Binarity of almost $\omega$-categorical quite o-minimal theories. Sberian Mathematical Journal 2020, 61:3, 379-390.

14. Kulpeshov, B.Sh. A criterion for binarity of almost $\omega$-categorical weakly o-minimal theories. Siberian Mathematical Journal 2021, 62:6, 1063-1075.

15. Sudoplatov, S.V. Classification of Countable Models of Complete Theories; NSTU: Novosibirsk, 2018.

16. Shulepov, I.V., Sudoplatov, S.V. Algebras of distributions for isolating formulas of a complete theory. Siberian Electronic Mathematical Reports 2014, 11, 380-407.

17. Sudoplatov, S.V. Algebras of distributions for semi-isolating formulas of a complete theory. Siberian Electronic Mathematical Reports 2014, 11, 408-433.

18. Sudoplatov, S.V. Algebras of distributions of formulas with respect to generalized semi-isolation. Algebra and Model Theory 9. Collection of papers. Eds.: A.G. Pinus, K.N. Ponomaryov, S.V. Sudoplatov, and E.I. Timoshenko; Edition of Novosibirsk State Technical University: Novosibirsk, 2013, 67-100.

19. Sudoplatov, S.V. Algebras of distributions for binary semi-isolating formulas for families of isolated types and for countably categorical theories. International Mathematical Forum 2014, 9:21, 1029-1033.

20. Sudoplatov, S.V. Forcing of infinity and algebras of distributions of binary semi-isolating formulas for strongly minimal theories. Mathematics and Statistics 2014, 2:5, 183-187.

21. Kulpeshov, B.Sh., Sudoplatov, S.V. On algebras of distributions of binary formulas for quite $o$-minimal theories. News of the National Academy of Sciences of the Republic of Kazakhstan. Physical-Mathematical Series 2015, 300:2, 5-13.

22. Emelyanov, D.Yu. On algebras of distributions of binary formulas for theories of unars. Bulletin of Irkutsk State University. Series Mathematics 2016, 17, 23-36.

23. Emelyanov, D.Yu., Kulpeshov, B.Sh, Sudoplatov, S.V. Algebras of distributions for binary formulas in countably categorical weakly o-minimal structures. Algebra and Logic 2017, 56:1, 13-36.

24. Emelyanov, D.Yu., Sudoplatov, S.V. On deterministic and absorbing algebras of binary formulas of polygonometrical theories. Bulletin of Irkutsk State University. Series Mathematics 2017, 20, 32-44.

25. Baikalova, K.A., Emelyanov, D.Yu., Kulpeshov, B.Sh, Palyutin, E.A., Sudoplatov, S.V. On algebras of distributions of binary isolating formulas for theories of abelian groups and their ordered enrichments. Russian Mathematics 2018, 62:4, 1-12.

26. Emelyanov, D.Yu., Kulpeshov, B.Sh, Sudoplatov, S.V. On algebras of distributions for binary formulas for quite o-minimal theories. Algebra and Logic 2018, 57:6, 429-444.

27. Emelyanov, D.Yu., Kulpeshov, B.Sh, Sudoplatov, S.V. Algebras of binary formulas for compositions of theories. Algebra and Logic 2020, 59:4, 295-312.

28. Palyutin, E.A., Saffe, J., Starchenko, S. S. Models of superstable Horn theories. Algebra and Logic 1985, 24:3, 171-210.

29. Sudoplatov, S.V. Syntactic approach to constructions of generic models. Algebra and Logic 2007, 46:2, 134-146.

30. Sudoplatov, S.V. Basedness of stable theories and properties of countable models with powerful types. Dis... cand. fiz.-mat. sc.: 01.01.06; Novosibirsk, 1990.

31. Shelah, S. Classification theory and the number of non-isomorphic models; North-Holland: Amsterdam, 1990.

32. Harnik, V., Harrington, L. Fundamentals of forking. Annals of Pure and Applied Logic 1984, 26:3, $245-286$.

33. Woodrow, R.E. Theories with a finite number of countable models and a small language. // Ph. D. Thesis; Simon Fraser University, 1976.

34. Baldwin, J.T., Lachlan, A. On strongly minimal sets. Journal of Symbolic Logic 1971, 36:1, 79-96. 